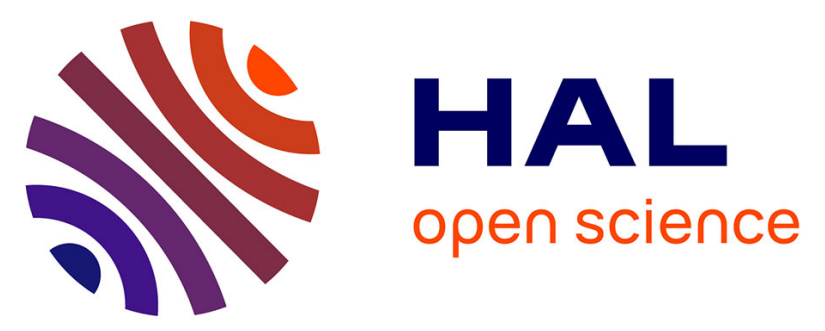

\title{
Particle charge fluctuation and its consequences on aerosol dynamics in dusty plasmas: from continuous to discrete statistical models
}

\author{
A. Michau, S Prasanna, S Longo, K. Hassouni
}

\section{To cite this version:}

A. Michau, S Prasanna, S Longo, K. Hassouni. Particle charge fluctuation and its consequences on aerosol dynamics in dusty plasmas: from continuous to discrete statistical models. Plasma Physics and Controlled Fusion, 2020, Special Issue Featuring the Invited Talks from the 46th EPS Conference on Plasma Physics, Milan, 8-12 July 2019, 62 (1), pp.014002. 10.1088/1361-6587/ab469f . hal-02394842

\section{HAL Id: hal-02394842 \\ https://hal.science/hal-02394842}

Submitted on 10 Dec 2019

HAL is a multi-disciplinary open access archive for the deposit and dissemination of scientific research documents, whether they are published or not. The documents may come from teaching and research institutions in France or abroad, or from public or private research centers.
L'archive ouverte pluridisciplinaire HAL, est destinée au dépôt et à la diffusion de documents scientifiques de niveau recherche, publiés ou non, émanant des établissements d'enseignement et de recherche français ou étrangers, des laboratoires publics ou privés. 


\title{
Particle charge fluctuation and its consequences on aerosol dynamics in dusty plasmas: from continuous to discrete statistical models.
}

\author{
A. Michau ${ }^{1}$, P. Swaminathan ${ }^{1}$, S. Longo ${ }^{2}$ and K. Hassouni, ${ }^{1}$ \\ ${ }^{1}$ LSPM, CNRS-UPR3407, Université Paris 13, 99 Avenue J. B. Clément 93430 Villetaneuse, \\ France \\ ${ }^{2}$ Department of Chemistry, University of Bari, Via E. Orabona 4, 70126 Bari, Italy. \\ E-mail: michau@1spm.cnrs.fr
}

Received xxxxxx

Accepted for publication $\mathrm{xxxxxx}$

Published xxxxxx

\begin{abstract}
In this article work we investigated different approaches and models used for the estimation of the charge fluctuation of solid particles in dusty plasmas. We compared the charge distributions inferred from these models and analysed the consequences in terms of coagulation kinetics and particle size distributions. We especially showed that taking explicitly into account the discrete nature of the charging process along with the enhancement of ion flux due to ion-neutral elastic collisions result in significantly larger values for the fractions of neutral and positively charged particles, which affects the coagulation kinetics and the predicted values of particle density. We also showed, by comparing sectional and modal approaches, that the typical size-distributions obtained in the studied plasmas may be well described using a bimodal distribution with a single size nucleation mode and a Gaussian large-size mode. Such a possibility enables a great simplification of the aerosol dynamic description and open up an interesting perspective as far as multidimensionnal self consistent modeling of dusty plasmas is concerned.
\end{abstract}

Keywords: dusty plasma, particle charging, nanoparticle

\section{Introduction}

Formation of solid particles may take place in non equilibrium plasmas under a variety of discharge conditions [1,2,3]. In some cases these (nano)particles are produced on purpose for their functionnal properties [4]. In other cases they are form accidentally produced and may be be detrimental for the targetted plasma process [1]. In both situations, whether one need to optimize or avoid the production of particles, it is necessary to understand the plasmas and aerosol dynamics processes involved in particle production [6]. In particular, particle coagulation seems to be a key process that governs the particle density and structures, and shapes the particle size-distribution [5]. It was early recognized that particle coagulation in discharge plasma can only take place if charge fluctuation enables the production of large enough amount of neutral or positively charged particles [10]. Surprisingly, the incorporation of this effect as a driver of the coagulation kinetics in dusty plasmas models were performed only quite recently by very few groups [7,14]. Most of the approaches used in dusty plasma modeling are based on a continuous statistical description of the charge fluctuation. This which assumes that, the charging beeing an ergodic Poisson process, the charge distribution is Gaussian [10]. This approach works fine as long as the mean charge value is much larger than the charge fluctuation magnitude, which is typically the case for large particles and large electron temperature discharge conditions [1]. It becomes questionable for small particles and/or low electron temperature conditions when the mean charge value is of the same order as the fluctuation magnitude. Analyzing the accuracy of this continuous statistical model and proposing 
other, hopfully more accurate, approaches to estimate the charge distribution for small particles is of prime importance as far as coagulation kinetics is concerned. Indeed, small particles are the only one that can show a large fraction of neutral or positively charged particles. Their charge distribution therefore governs the coagulation kinetics. The first objective of this paper is related to this last point. More specifically, we compare the charge distributions inferred from a continuous and discrete statistical models for particle of different sizes. We investigate how expliciteley taking into account the discrete nature of the Poisson charging process in a dusty plasma model affect the predicted particle size distributions. We also analyse how the charging processes is affected by the ion current enhancement due to ion-neutral elastic collisions in the torr regime.

In all these studies we describe the aerosol dynamics using a sectional model that enables predicting the size distribution without any assumption and with a good accuracy. This approach has the drawback to present a relativeley important complexity. In particular, it can be hardly used in multidimensional dusty plasma models. Therefore, based on the results obtained using the sectional approach, we proposeinvstigate, in the second part of the paper, an alternative models that makes use of a modal approach in order to determine the typical bimodal size-distributions obtained in the investigated dusty plasmas.

The paper is organized as follows: section 2 gives a brief description of typical discharges considered in this work, section 3 presents the different charging models as well as a sensitivity study of the predicted charge and particle disctributions to these models, section 4 presents the simplified modal approaches used to describe the aerosol dynamics and determine the bimodal size distributions that are typically obtained in the investigated plasma, section 5 gives a brief summary of the results and discusses some research perspectives that may be drawn from this work.

\section{Brief description of the studied Plasma Discharges and of the dusty plasma model used for their investigation}

\subsection{Studied plasmas discharges}

In this study, we consider a sputtering argon DC discharge with a graphite catode. This that discharge has been extensively used to produce carbon nanoparticles [5]. The inter-electrode distance is $14 \mathrm{~cm}$, the pressure is $60 \mathrm{~Pa}$ and the discharge current is set to $80 \mathrm{~mA}$. The graphite cathode is sputtered by fast ions and neutrals, which produces carbon atoms and small amounts of $\mathrm{C}_{2}$ and $\mathrm{C}_{3}$ clusters. These clusters undergo molecular aggregation, attachment and charge transfer processes which results in the nucleation of solid particles. These nanoparticles grow up to $\sim 40 \mathrm{~nm}$ diameter by molecular deposition of clusters on their surface and coagulation [5].

\subsection{Numerical Modelling}

A dDusty plasma model [6] was previously developped to describe this discharge. It consists of three modules describing (i) the DC discharge dynamic equilibrium, (ii) the molecular growth of clusters and (iii) the aerosol dynamics. The last module used the so-called sectional model that accounts for a large number of size bins (or 'sections') over 1-100 nm size domain along with a charge distribution for each size bin [7]_[8]. The advantage of this kind of model is to accuratly take into account the influence of the particle size on the non-linear aerosol dynamic processes. The counterpart of this precision accuracy is the numerical expense which makes it difficult to extend this approach to very large, 2D or 3D simulations.

The developped one-dimensionnal dusty plasma model have shown good agreement with experimental results, especially in the description of the particle size distribution [9]. In particular we showed that particle formation and growth is only possible in the elctric field reversal region where negative species are trapped. We also showed that the particle nucleation takes place during the whole discharge duration so that the particle size distribution is controlled by the combination of three processes : nucleation, surface sticking and coagulation. Typical distributions show high particle densities for the small size domain, just above the nucleus size, and the large size domain that determine the mean diameter. Intermediate size particles are highly depleted which were attributed to coagulation. In other words, coagulation is responsible for the creation of two modes in the particle size distribution : a nucleation mode and a core distribution mode. All the aerosol dynamics phenomena, i.e., transport, nucleation, coagulation, surface-sticking of clusters, are controlled by the particle charge and more precisely charge fluctuation. We described the charge fluctuation dynamic by assuming that the charging process is ergodic in nature and by using a charge distribution as suggested by Matsoukas [10]. 
In the previous version of the model, following Matsoukas et al [10], we made use of a continuous statistical approach and assumed that the Poisson charging process results in a gaussian charge distribution. The standard deviation of this distribution depends on the plasma parameter and particle size. It determines the charge fluctuation and the fraction of neutral and even positively charged particles that can play a key-role in the growth mechanism, especially through coagulation with the negatively charged particles of the core distribution [14].

\section{Particle Charging Model: sensitivity studies}

In Matsoukas et al approach [10] the mean charge of a given particle is obtained assuming OML equilibrium ( $\left.j_{e, \dot{q}}=j_{i, \dot{q}}\right)$ and the charge distribution obtained on the basis of continuous statistics is however valid for large particles that carry a significant number of elementary charges, in which case the charge fluctuation magnitude is small compared to the mean-value of the particle charge. This becomes questionable in the case of small particles or/and moderate electron temperature plasmas when, the charge fluctuation magnitude is comparable to the mean value of the charge carried by the particles. In this case, the charge fluctuation phenomenon cannot be described using a continuous charge distribution and the discrete nature of the charging processes has to be explicitly taken into account.

For this purpose, we first developed a discrete OML model for particle charging [11]. In this model, the charge distribution is discretised using a set of integer charge states that expand over a large enough charge-domain. The discrete Poisson charging processes is solved for each charge state in terms of the normalized charge population, $\phi_{q}$, at charge q. The dynamic of $\phi_{q}$ depends on the electron, $\mathrm{j}_{\mathrm{e}, \mathrm{q}}$, and ion $\mathrm{j}_{\mathrm{i}, \mathrm{q}}$, currents collected by the particles of a surface $S_{p}$ following the expression:

$$
\begin{gathered}
j \\
\frac{1}{S_{p}} \frac{d \varphi_{q}}{d t}=j_{e, q+1} \varphi_{q+1}+j_{i, q-1} \varphi_{q-1}-
\end{gathered}
$$

Usually for the particle size considered in this work, i.e., 10-100 nm, the electron and ion currents are inferred from the OML theory assuming a collisionless sheath. Recent A recent work by Chahl and Gopalakrishnan [12] shows however that ion-neutral elastic collisions may be significant enough to increase the ion capture section and to enhance the ion collection current even at fairly low pressure. We therefore also considered this collision-induced ion current enhancement and analysed its impact on the charging kinetics and charge fluctuation.

Figures 1 shows the charge distribution calculated for $1 \mathrm{~nm}$ (a) and 10nm particle (b) using using the continuous charging model assuming OML theory [10], the discrete charging statistical model (Eq 1) either assuming collisionless charging model (denoted discrete) or taking into account the collision-induced enhancement of ion collection current (denoted CG).

It appears that the three charging models are in good agreement for large particles (10 nm diameter) even-though the charging level, with a mean value of 2 , is fairly low. The situation is different in the case of small particles, i.e. $1 \mathrm{~nm}$ diameter for which the three charging models give significantly different results. In particular, the mean charge-value, below one elementary charge, is smaller than the charge fluctuation amplitude.

The detailed charge distribution, especially the fraction of neutral and positively charged particles, plays a key-role in the growth process of the particle since collisions with negative species, i.e., coagulation with the dominant large negative particle or surface deposition of negative cluster, are only effective for non-negatively charged small particles. 

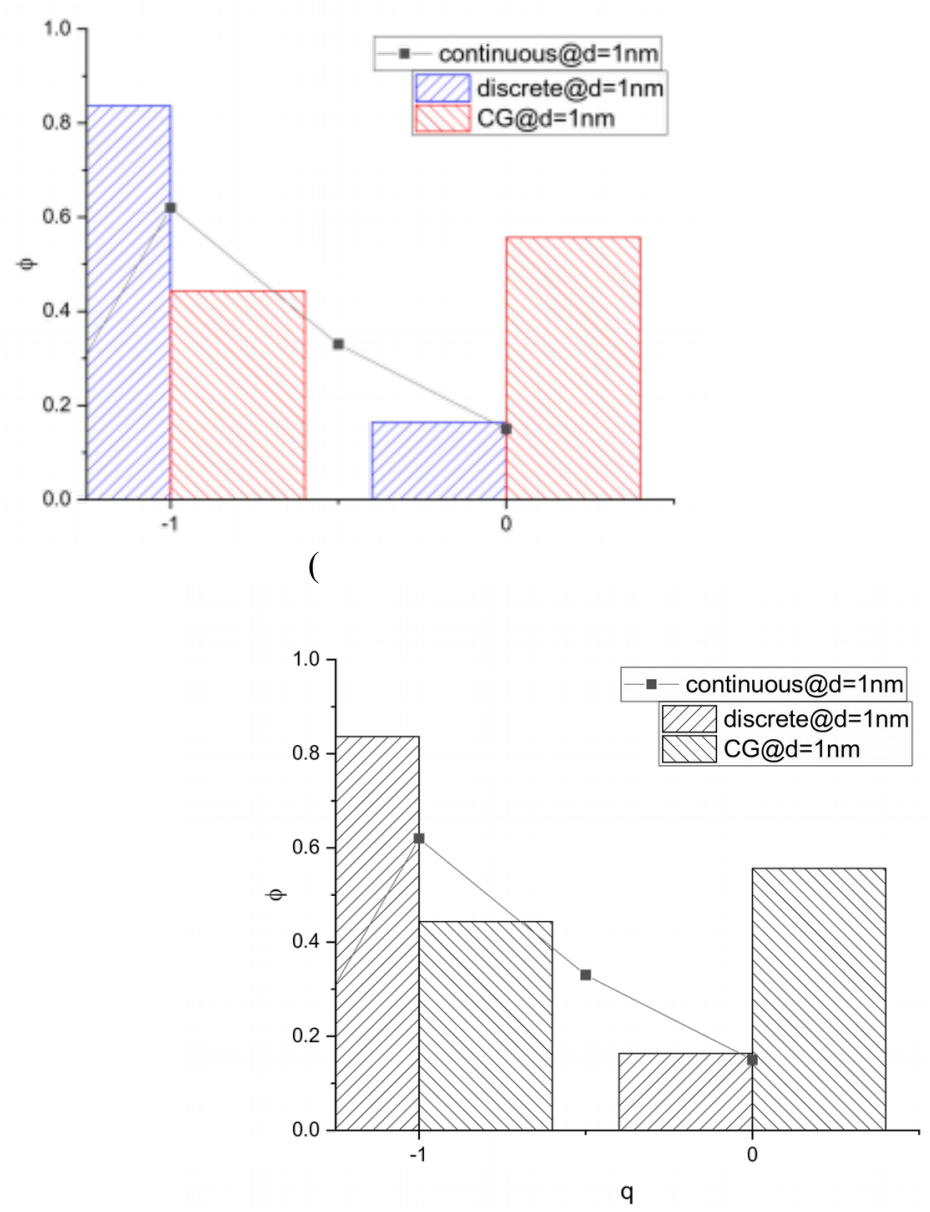

(a)
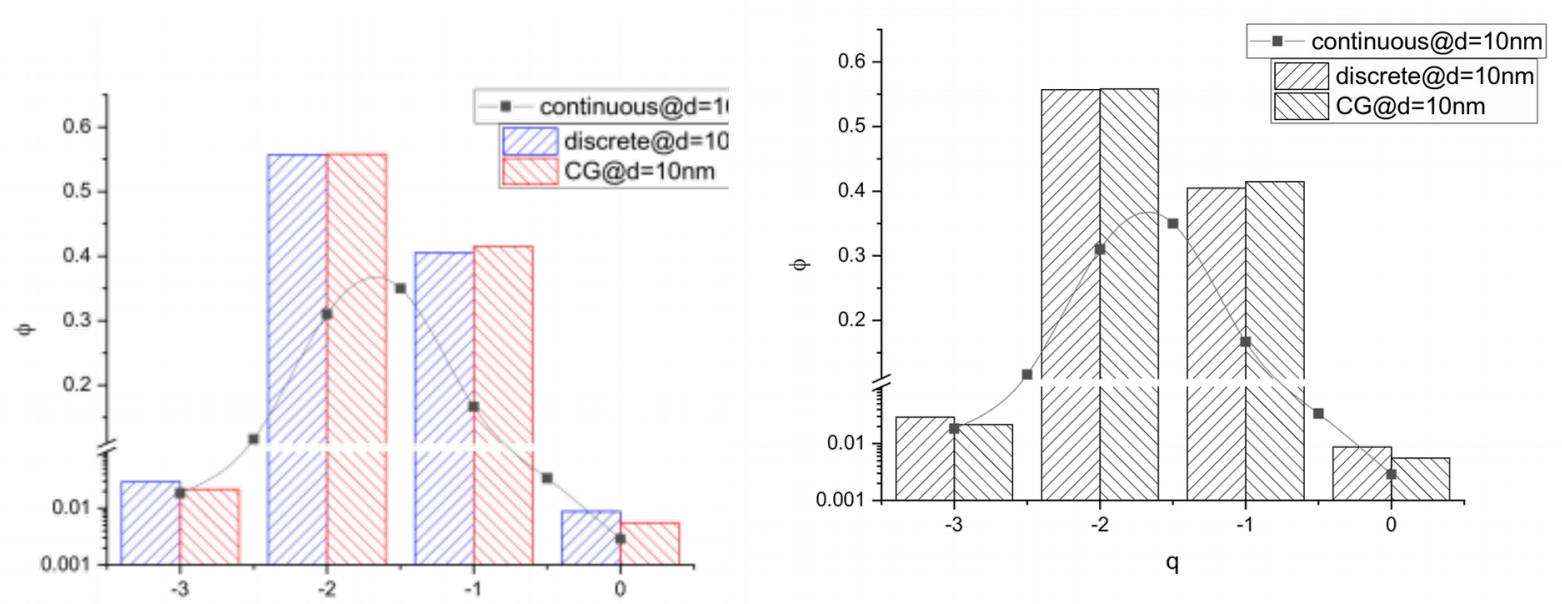

(b)

Figure 1 : Calculated Normalized charge distributions for $1 \mathrm{~nm}$-size (a) and $10 \mathrm{~mm}$-size (b) particles using the continuous statistical model proposed by Matsoukas [10] (continuous), the discrete statistical collision-less model (discrete) and the discrete statistical collisional model (CG). The discharge conditions are those of reference [5]. The cold electron density and temperature used in the simulation corresponds to the value obtained at the field reversal, i.e., $n_{e}=10^{11} \mathrm{~cm}^{-3}$ and $T_{e}=0.1[14]$.

Figure 2 depicts the fraction of non-negative particles as a function of the particle diameter computed with the different charging models. It appears that continuous and discrete collisionless charging models yield similar values for the fraction of non-negative particles with size below $5 \mathrm{~nm}$. However, the continuous model significantly underestimates the population of non-negative particles with diameter values above $5 \mathrm{~nm}$. In particular, the discrete model yields a nonnegative particle fraction above $1 \%$ up to $10 \mathrm{~nm}$ size particles. On the opposite, the continuous model predicts a very 
sharp depletion, i.e. more than two orders of magnitude, of the non-negative particle fraction in the size range between 5 and $7 \mathrm{~nm}$. On the other hand, the discrete collisional charging model predicts larger amount of neutral particles as compared to the collisionless model for sizes below $5 \mathrm{~nm}$. The fraction of non-negative particle with sizes greater than 5 $\mathrm{nm}$ predicted by this model is however significantly smaller than that determined assuming collisionless discrete charging model, i.e., almost one order of magnitude for a $10 \mathrm{~nm}$ diameter.

We implemented these different charging models in the aerosol module of the 1D dusty plasmsa model discussed in reference [14] and [2] and [14]. Since our aims is mainly to analyse the sensitivity of aerosol dynamics to the adopted charging model, the simulation performed and discussed in this article are not fully self consistent. The discharge conditions and cluster dynamics are taken from previous simulation [14] and only the aerosol module is solved in order to emphasize the effect of the charging model on particle growth.
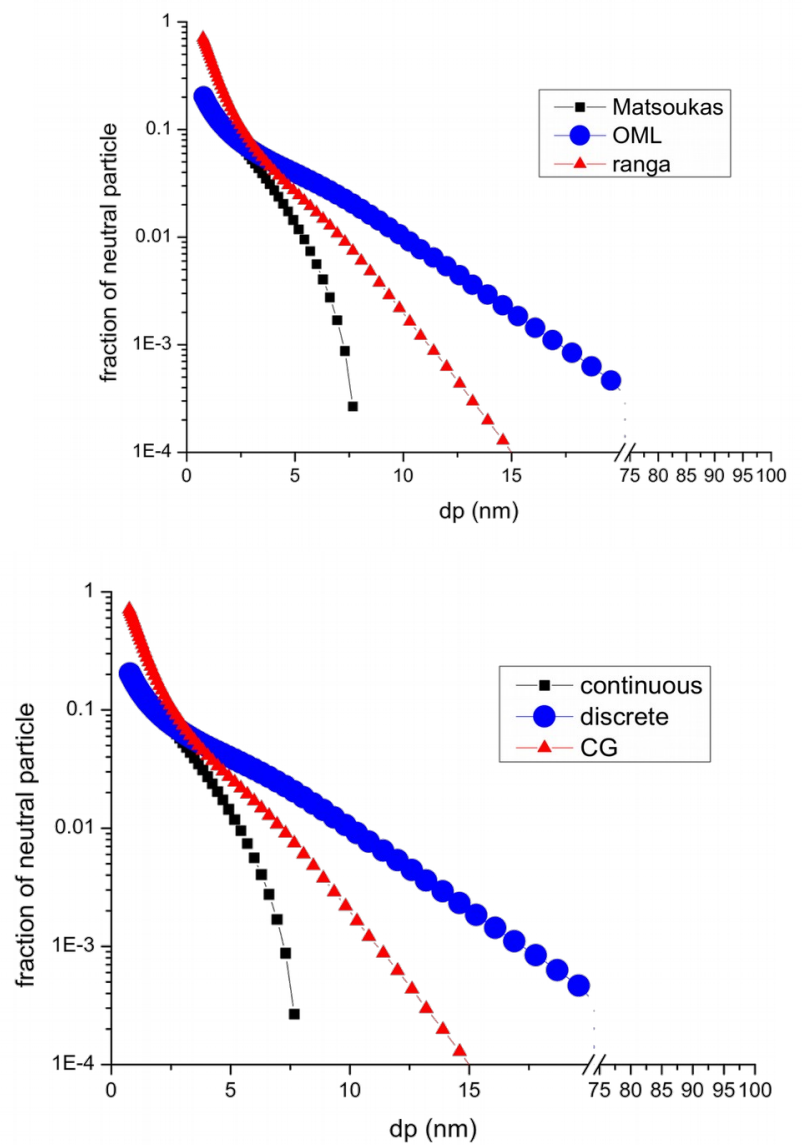

Figure 2 : Calculated non-negative fractions of dust particle as function of their size using the continuous statistical model proposed by Matsoukas [10] (continuous), the discrete statistical collision-less model (discrete) and the discrete statistical collisional model (CG). The discharge conditions are those of reference [5]. The cold electron density and temperature used in the simulation corresponds to the value obtained at the field reversal, i.e., $n_{e}=10^{11} \mathrm{~cm}^{-3}$ and $T_{e}=0.1$ [14]

Figrure 3 depicts the time-evolutions of the total density and mean-diameter of the particles at the maximum density position (the field reversal position 2).

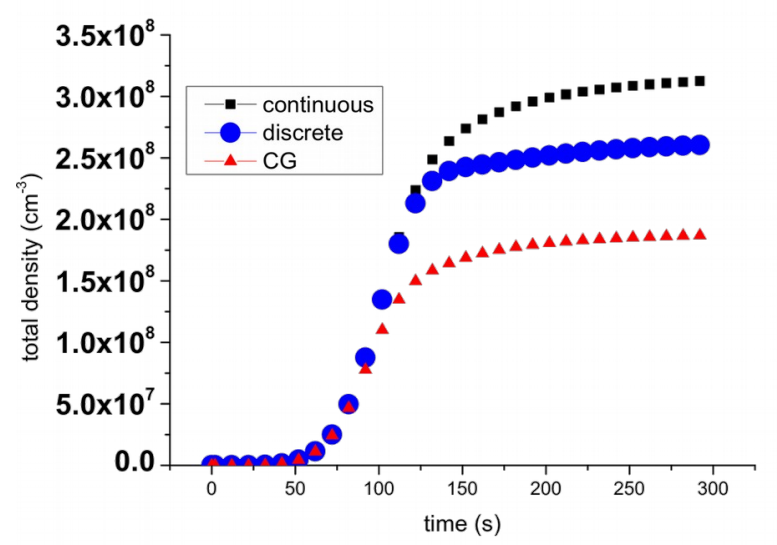




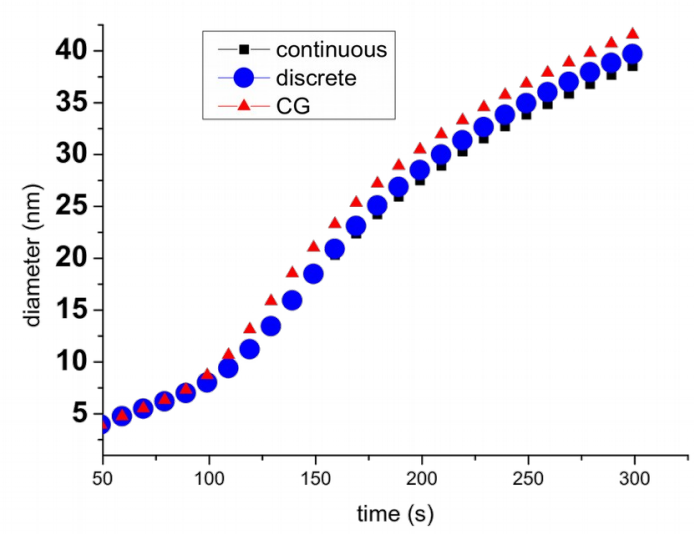

Figure 3: Time evolutions of the total density and mean diameter of the produced dust particles calculated using the continuous statistical model proposed by Matsoukas [10] (continuous), the discrete statistical collision-less model (discrete) and the discrete statistical collisional model (CG). The discharge conditions are those of reference [5]. The cold electron density anddensity and temperature used in the simulation corresponds to the value obtained at the field reversal, i.e., $\mathrm{n}_{\mathrm{e}}=10^{11}$ $\mathrm{cm}^{-3}$ and $\mathrm{T}_{\mathrm{e}}=\mathbf{0 . 1}[14]$

The three models show similar trends. Nucleation starts around $50 \mathrm{~s}$ after the discharge ignition. It causes a steep increase of the particle density until $\sim 150 \mathrm{~s}$ time-period. Then, the density remains almost constant, while the mean diameter keeps increasing monotonically at a rate of approximately $\sim 10 \mathrm{~nm} /$ min during the whole discharge phase.

Both the collisionless and collisional discrete charging models yield an increased mean diameter and a reduced total density as compared to the continuous OML charging model. This result is a consequence of the larger coagulation rate induced by the enhanced fraction of neutral particles that is obtained when discrete charging model is used.

Figure 4 presents the obtained particle size distributions for the considered charging models for plasma durations of 100 s and 300s.
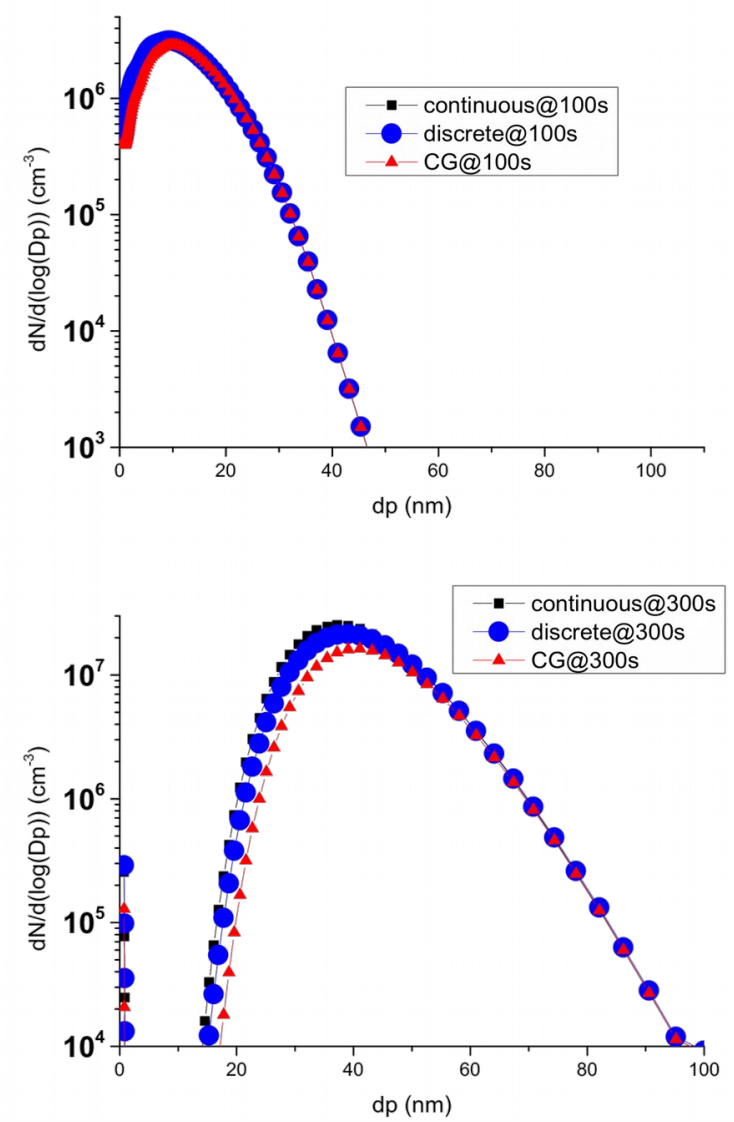

Figure 4 : Particle size distributions obtained from the dusty plasma model, at the maximum electron density position for discharge durations of $100 \mathrm{~s}$ (a) and $300 \mathrm{~s}$ (b), using the continuous statistical model proposed by Matsoukas [10] (continuous), the discrete statistical collision-less model (discrete) and the discrete statistical collisional model (CG). The simulated discharge conditions are those of reference [14]. 
The three models show similar time evolution of the size distribution : these are monomodal distributions for time duration below $150 \mathrm{~s}$ and becomes bimodal above. The bimodal distributions is are composed of a first very narrow mode in the size range close to the diamater of the smallest nucleus, i.e. $1 \mathrm{~nm}$, and a much wider second mode with a log-normal distribution shape. Continuous and discrete OML yield similar estimates for the particle density of the nucleation mode. This is not the case for the core distribution mode, the population of which, is overestimated by the continuous charging model. This difference is due to the larger fraction of the non-negative particles and the subsequent enhanced coagulation kinetics predicted by the discrete model.

On the other hand, the density values predicted using the collisional discrete (CG) model for both the nucleation and the large size modes are smaller than those obtained with the other charging models. This model therefore yields an enhanced coagulation kinetics and larger values of the mean diameter as compared to the other models. This result is explained by the largest value predicted for the fraction of neutral small particles contained in the nucleation mode by the collisional discrete $(\mathrm{CG})$ model.

\section{Simplified approach for aerosol dynamics modelling}

The simulations discussed in the previous sections are based on a discrete sectional representation of the particle size distribution. This method has two advantages: it does not require any assumption on the shape of the particle size distribution and it shows a very good accuracy. This method It requires however a high size resolution to minimize the numerical diffusion that can alter the accuracy of the numerical approximation of the molecular deposition flux term in the aerosol dynamic equation [13]. The numerical cost associated with this high accuracy makes this approach barely feasible for large dusty plasma simulations, and one has to look for simplified approaches. Since, the particle size distribution obtained in the investigated plasmas always show one or two log-normal modes [4], it is possible to adapt a parametrized model based on log-normal mode size distributions originally developped for air armospheric aerosol systems to simplify the description of the aerosol dynamics in our plasmas [5]. In this approach, the size distribution of each mode is approximated by an analytical log-normal distribution which can be completely fully described by three parameters, namely the particle density in the mode $(\mathrm{Ni})$, the average particle diameter (dpi) and the standard deviation ( $\sigma i)$ for each mode. The three distribution parameters can be determined by solving a combination of three moments, M0, M3, and M6, of the General Dynamics Equation. A charging dynamics equation (Eq 1) has to be solved for each mode.

For each mode, the dynamic equation takes into account particle transport, particle nucleation, particle growth by deposition of molecular species and coagulation processes either between two particles belongings to the same mode, i.e. intra-modal coagulation, or between particle belongings to different modes, i.e. inter-modal coagulation [15]. However, in this so- called Whitby's model, the modes that characterize the distribution have to be prescribed $a$ priori. The major consequence of this, is that the appearance of a distribution mode during the growth process cannot be accounted for. Since in our case the larger mode appears only $150 \mathrm{~s}$ after the discharge ignition, we extended Whitby's model to account for the creation of new particle in the larger mode from intra-modal coagulation. To treat this issue, Megaridis and Dobbins [6] proposed a modeling approach for bimodal distribution where coagulation processes occuring in the small size mode yield a full particle transfer to the larger size mode, i.e., the core distribution mode. We also therefore adapted this approach to our situation by assuming that coagulation between particles belonging to the nucleation mode results in a particle in the large-size mode, i.e., the core distribution mode.

Figure 5 shows the particle size distributions obtained in the same conditions as discussed above and for $300 \mathrm{~s}$ discharge duration using either the sectional model, the modal model without transfer, i.e., Whitby's model [15], and a the modal model with full transfer by coagulation from the nucleation mode to the core distribution mode, i.e., Megaridis model [16]. The results clearly show that bimodal distribution cannot be obtained when no transfer is considered between the two distribution modes. Whistby's model is in particular not able to predict the presence of the nucleation mode, which leads to the underestimation of the coagulation kinetics.

We also notice that the particle size distribution predicted by Withby's model shows slightly higher density and lower mean diameter as compared with the distribution obtained from the sectional model. 


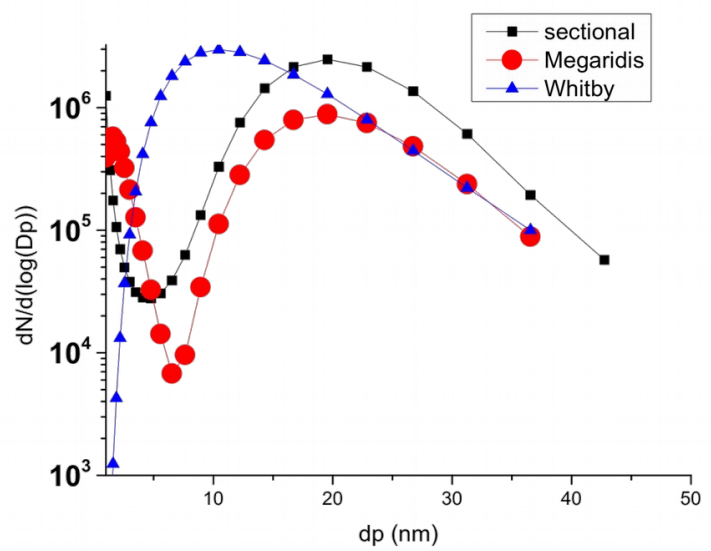

Figure 5: Particle size distributions obtained from the dusty plasma model, at the maximum electron density position and for discharge durations of at $300 \mathrm{~s}$, using the sectional model, Whitby's bimodal approach and Magaridis's bimodal model. The simulated discharge conditions are those of reference [14].

Figure 5 also shows that Megaridis model is capable of capturing the bimodal shape of the distribution. However, the nucleation mode predicted by this model shows much larger density over a larger size domain as compared with the one determinedde by the sectional model. This results is consistent with the much smaller density value obtained for the core distribution mode when using Megaridis model. This result It is at least partly due to a significant underestimation of the rate of inter-mode particle transfer through the growth by cluster deposition on particle surface. The evaluation of this transfer rate in Megaridi's is not straitforward since it requires defining relevent size criteria that may be used to evaluate intermode trasnfer transfer kinetics through cluster deposition on particle surface.

As a final alternative, we also analyzed a simplified bimodal approach proposed by Jeong et al [7]. In this case, the nucleation mode is approximated by a single size distribution with a diameter-value that corresponds to the size of smallest solid nucleus, while no approximation is used for the larger mode. Therefore, all the growing particle of the nucleation mode are transfered to the larger mode. This model is therefore able to take into account the key-role of the nucleation mode in the coagulation process with a much simpler mathematical formulation as compared to Whitby's and Megaridi's models. Further, since neutral and positively charged particles produced by charge fluctuation essentially belong to the nucleation mode, the particles belonging to the large size mode being always negatively charged, the coagulation process can be accurately described by taking into account the charge fluctuation for the only nucleation mode.

Figure 6 shows the particle size distributions obtained at $300 \mathrm{~s}$ with the sectional model and the bimodal model proposed by Jeon et al. [17]. It appears that the simplified approach, yields a good estimates for both the density of the nucleation mode and the size distribution of the large size mode. The only difference resides in the fact that the large size distribution mode obtained by the sectional model is larger than the one inferred from the simplified modal approach. This is probably due to the fairly different treatment of particle growth kinetics by surface-deposition of clusters. This effect is indeed considered for each bin in the sectional model which therefore accounts for the enhancement of this growth route for the larger sizes, i.e., the growth rate is a linear function of the surface. This effect also explains the asymmetric shape of the large size distribution obtained from the sectionnal model, i.e., the core distribution is more populated above the mean dimater than below. The large size distribution obtained using the bimodal approach of Jeon et al. exhibits a less asymmetric shape. Note however, that the shape asymmetry observed when the sectional approach is at least also partly due to the numerical diffusion that appears in the numerical approximation of the aerosol dynamic equations when stable non-symmetric upwinding is used to evaluate the convective growth flux due to surface-deposition of clusters. We believe therefore that the 'actual' large size distribution would be somewhat between the distributions destermined by the sectional model and by the modal approach of Jeon et al. In any case, the particle density-values and the partice mean-diameters obtained by the two approaches are in good agreement. 


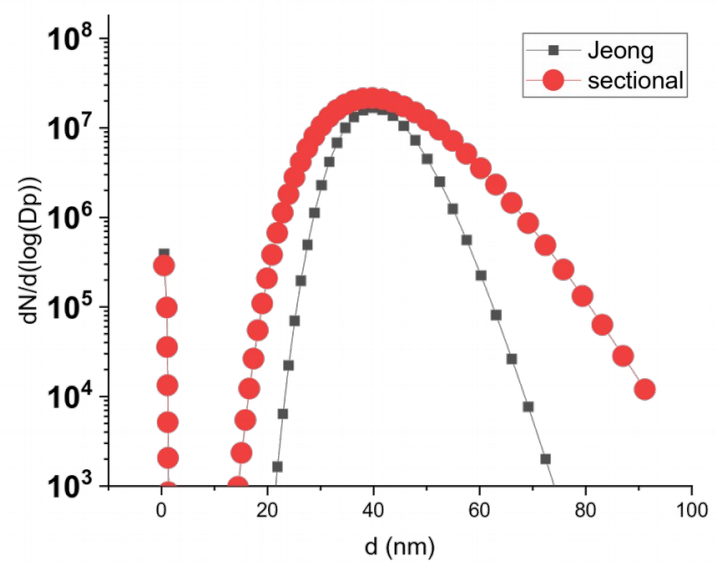

Figure 6 : Particle size distributions obtained from the dusty plasma model, at the maximum electron density position and for discharge durations of at $300 \mathrm{~s}$, using the sectional model and Jeong's bimodal model. The simulated discharge conditions are those of reference [14].

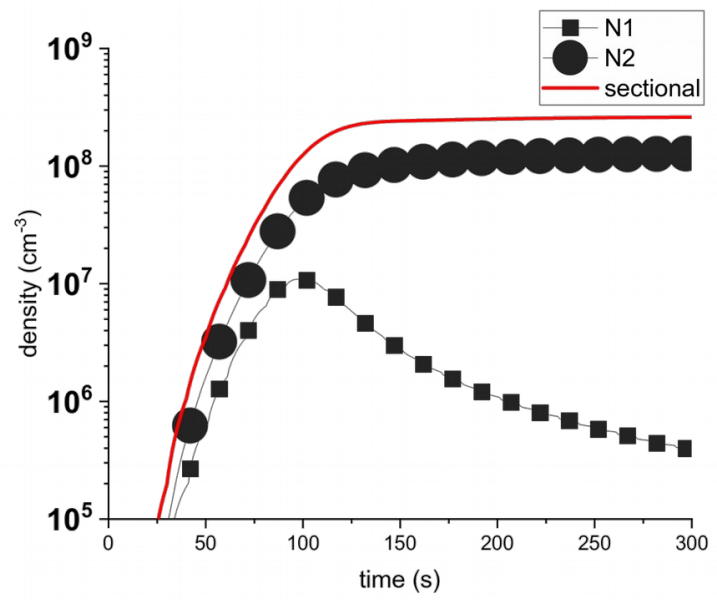

(a)

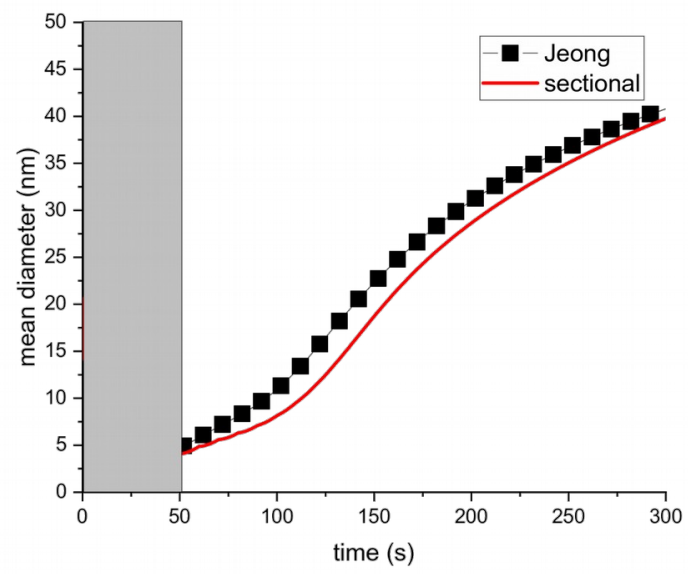

(b)

Figure 7 : Time-evolution of particle densities (a) and mean diameter (b) obtained from the dusty plasma model, at the maximum electron density position, using the sectional model and Jeong's bimodal model. The densities of both the nucleation and core distribution modes are given in the case of Jeong's model. The simulated discharge conditions are those of reference [14].

This may be clearly seen Figure 7 that shows the time-evolution of the number density of for both nucleation and large size modes obtained from Jeong's model along with the total particle density obtained from the sectional model. The particle density of the core distribution obtained from Jeong's and sectional models are in good agreement. They show 
very similar time-evolutions. The time-evolution of the nucleation mode density cleary reveals the different regimes that take place during particle gowth. Between $50 \mathrm{~s}$ and $100 \mathrm{~s}$ we see a similar density-values for both modes, which is indicative of unimodal distribution. For time periods greater than $100 \mathrm{~s}$, there is a decrease of the density of the particles belonging to the nucleation mode that are consummed in the coagulation with larger particles. This is consistent with the bimodal nature of the distribution shown in Figure 6.

Notwithstanding its limitations, Jeong's modal model appears to be the best alternative to the sectional model. It yields the distribution parameters with fairly good accuracy, requires a low computational cost and makes use of relatively simple mathematical expressions for the closure terms of moments and charge fluctuation.

\section{Conclusion}

The modeling study performed in this work clearly shows that it is necessary to explicitly taking take into account the discrete nature of the charge fluctuation along with the collisional ion current enhancement of ion current as far as charge fluctuation evaluation is concerned. The fraction of neutral and positiveely charged particles are is significanty enhanced by theses effects. This in turn leads to a significant enhancement of the coagulation kinetics, which results to a threefold decrease of the particle density. We also showed that a simplified method originally developped for modeling atmospheric aerosol dynamic may be well adapted to describe the bimodal particle size-distributions that are typically obtained in non equilibrium plasmas.

By validating fairly straightfirward straightforward and useful approaches that enables accurately describing the charge fluctuation for the small particles and predicting the bimodal nature of the particle size distribution, the present work open up interesting perspectives as far as multidimensional fully self consistent dusty plasma model development is concerned.

\section{Acknowledgements}

\section{References}

[1] L Boufendi, M Ch Jouanny, E Kovacevic, J Berndt, M Mikikian. Dusty plasma for nanotechnology.Journal of Physics D: Applied Physics, IOP Publishing, 2011, 44 (17), pp.174035.

[2] C Arnas et al 2010 Plasma Phys. Control. Fusion 52124007

[3] Hassouni, Khaled, Francis Mohasseb, Fabien Bénédic, Guillaume Lombardi and Alix Gicquel. "Formation of soot particles in Ar/H2/CH4 microwave discharges during nanocrystalline diamond deposition: A modeling approach." Pure and Applied Chemistry 78 (2006): 1127 - 1145.

[4] Mariotti, D. , Belmonte, T. , Benedikt, J. , Velusamy, T. , Jain, G. and Švrček, V. (2016), Low-Temperature Atmospheric Pressure Plasma Processes for “Green” Third Generation Photovoltaics. Plasma Process. Polym., 13: 70-90. doi:10.1002/ppap.201500187

[5] C. Arnas, C. Dominique, P. Roubin et al., "Experimental study of different carbon dust growth mechanisms," Journal of Nuclear Materials, vol. 337-339, pp. 69-73, 2005.

[6] A. Michau, K. Hassouni, C. Arnas et al., "On the Competition Between the Phenomena Involved in the Aerosol Dynamics in Sputtering Nonequilibrium Plasma," IEEE Transactions on Plasma Science, vol. 44, no. 4, pp. 528-534, 2016.

[7] F. Gelbard, Y. Tambour, and J. H. Seinfeld, "Sectional representations for simulating aerosol dynamics," Journal of Colloid and Interface Science, vol. 76, no. 2, pp. 541-556, 1980.

[8] P. Agarwal, and S. L. Girshick, "Sectional modeling of nanoparticle size and charge distributions in dusty plasmas," Plasma Sources Science and Technology, vol. 21, no. 5, pp. 055023, 2012.

[9] A. Michau, C. Arnas, G. Lombardi et al., "Nanoparticle formation and dusty plasma effects in DC sputtering discharge with graphite cathode," Plasma Sources Science and Technology, vol. 25, no. 1, pp. 015019, 2016.

[10] T. Matsoukas, and M. Russell, "Particle charging in low-pressure plasmas," Journal of Applied Physics, vol. 77, no. 9, pp. 4285-4292, 1995. 
[11] S. Prasanna, A. Michau, K. Hassouni et al., "Effect of charge fluctuation on nanoparticle heating in dusty plasma," Plasma Sources Science and Technology, vol. 28, no. 3, pp. 03LT03, 2019.

[12] H. S. Chahl, and R. Gopalakrishnan, "High potential, near free molecular regime Coulombic collisions in aerosols and dusty plasmas," Aerosol Science and Technology, pp. 1-27, 2019.

[13] C. Seigneur, A. B. Hudischewskyj, J. H. Seinfeld et al., "Simulation of Aerosol Dynamics: A Comparative Review of Mathematical Models," Aerosol Science and Technology, vol. 5, no. 2, pp. 205-222, 1986/01/01, 1986.

[14] A. Michau, C. Arnas, and K. Hassouni, "Aerosol dynamics in a sputtering DC discharge," Journal of Applied Physics, vol. 121, no. 16, pp. 163301, 2017.

[15] E. R. Whitby, and P. H. McMurry, "Modal Aerosol Dynamics Modeling," Aerosol Science and Technology, vol. 27, no. 6, pp. 673-688, 01/01

2011/09/29, 1997.

[16] C. M. Megaridis, and R. A. Dobbins, “A Bimodal Integral Solution of the Dynamic Equation for an Aerosol Undergoing Simultaneous Particle Inception and Coagulation," Aerosol Science and Technology, vol. 12, no. 2, pp. 240-255, 1990/01/01, 1990

[17] J. I. Jeong, and M. Choi, "A bimodal moment model for the simulation of particle growth," Journal of Aerosol Science, vol. 35, no. 9, pp. 1071-1090, 2004. 